\title{
The role of the 'specialist' in healthcare
}

\author{
Mark Britnell
}

The role of the specialist in healthcare has never been in doubt and its prominence is increasing.

In this article, I'm going to argue that specialisation is needed to improve our national competitiveness, ability to innovate, extend life and explore the frontier of science and knowledge. I'm also going to argue that, if left unfettered and not harnessed to the role of the generalist in healthcare, we can look forward to painful debates about rationing, professional 'turf-war' and suboptimal patient care. I conclude that better teamwork between the generalist and specialist has never been more important for the NHS and doctors should get together to form 'integrated care chambers' to better serve their patients and the population at large, thus changing the pattern of healthcare that has built up over centuries.

In his documentary Civilisation, Professor Niall Ferguson argues that the West became the dominant global powerhouse from the 16th century because of the simultaneous combination of six 'killer applications' - competition, science, democracy, medicine, consumerism and the work ethic. Given current political debate about NHS privatisation, it is interesting to note that medicine and science have always helped British competitiveness and capitalism. Throughout the 16th century, it would have been impossible for the West to assert its growing economic supremacy without a strong medical and scientific base. The pursuit of knowledge in these fields has acted as a powerful economic spur and this is reflected in the UK's 21 st century desire to lead on innovation and promote our science and academic medical base throughout the world. The West has dominated global development for the past five centuries and will need to use innovation in science and medicine to a much greater degree if it is to maintain its international competiveness. Specialisation provides both the impetus and spur to maintain this dynamic force.

Indeed, it was Henry VIII in the 16th century that began the modernisation and specialisation of healthcare in England. Poor relief was becoming increasingly problematic during the early part of the century due to a rising population (from two million in 1485 to nearly three million by 1509), and the dissolution of the monasteries from 1537 effectively closed the majority of hospitals that were run by monks and considered to be special types of religious houses that were 'more concerned with saving souls than lives'. Ironically therefore, the dissolution of the monasteries paved the way for the workhouse (where the generalists started) and stimulated the introduction of hospitals which were, over time, associated with specialist care. It is no

Mark Britnell, chairman and partner, Global Health Practice, KPMG LLP coincidence that the rise of the royal college movement started in this century, so the origins of specialisation have long historical and cultural roots.

Specialisation is a trend that cannot be stopped. The average GP will receive about $15 \mathrm{~kg}$ of new clinical guidelines each year, while the number of articles captured and indexed annually by the electronic MEDLINE database stands at around 900,000. ${ }^{1}$ The number of identifiable diseases and their related treatments have increased manifold - the current World Health Organization International Classification of Diseases (ICD-10) currently stands at 155,000 codes. It is not humanly possible for individuals to master all this knowledge and information and, therefore, a degree of specialisation is both important and inevitable. Even a recent House of Lords Science and Technology Committee report ${ }^{2}$ found that healthcare professionals are currently not well equipped to use genomic tests effectively and interpret them accurately, indicating an urgent need for much wider education of healthcare professionals and the public in genomic medicine. That is before the generalist is required to master the implications of nanotechnology, pharmaceuticals and new biochemical pathways!

There are, of course, good clinical arguments for specialisation which we should acknowledge. Firstly, procedures have become more complex and effective. Percutaneous primary coronary intervention (PPCI), previously known as angioplasty, requires specialist training, specialist equipment and a specialist centre. In 2009-10, the Myocardial Ischaemia National Audit Programme (MINAP) reported that the use of PPCI had exceeded that of thrombolytic treatment for the first time. ${ }^{3}$ Surgery has also experienced significant levels of specialisation and the general surgeons of old have been superseded by vascular, endocrine, oncological and gastrointestinal subspecialties, to name but a few.

Secondly, specialisation produces better outcomes for patients. The relationship between volume and outcome has been well documented for some time as the recent 'media storm' over paediatric cardiac surgery indicates. The European Association for Cardio-Thoracic Surgery Congenital Heart Committee has advised that a minimum threshold of procedures be undertaken each week if specialist skills are to be maintained, while Sir Bruce Keogh, NHS medical director and former cardiac surgeon, has called for a minimum number of four surgeons working together to carry out at least 400 operations per year. ${ }^{4,5}$ KPMG is currently supporting the Department of Health in its review of the number of specialist sites that should be accredited to undertake this care in the future. It is commonly accepted in studies of paediatric cardiac surgery that the association between volume and in-hospital mortality is strong; as the 
complexity and risk of the procedure increases, and the more experience surgeons gain in their specific field of expertise, the better quality of care delivered. Of course, this places further pressure on doctors in training to specialise sooner rather than later thus generating more resource competition and pressure between the generalist and specialist.

Thirdly, and most importantly, patients themselves will drive further specialisation. As consumers become more informed, they will demand the latest, best and most timely diagnosis and treatments available. Through the explosion of the internet, patients now have more access to clinical information than ever before; eight in 10 internet users research health literature online and increasingly demand more from their care professionals. As outcomes on clinical quality become more available and better understood, patients will choose specialist centres which offer better outcomes. The NHS already has NHS Choices and the Good hospital guide, how long before we get the Which? of health?

Finally, specialisation can be cost effective. Professor Michael Porter of Harvard Medical School argues that the medical workforce of the future will be organised around the needs of the patient rather than traditional organisational boundaries. ${ }^{6} \mathrm{He}$ argues that care will be increasingly focused on outcomes, financed through bundled payments for the full care of a patient's medical problem, enabled through sophisticated IT systems that share knowledge and clinical details both quickly and seamlessly. Porter's vision reflects the need for increased specialisation which is organised on an efficient and effective scale. As the National Paediatric Surgery Review concluded in February 2011 'specialist equipment, used during surgery itself (eg lasers) or post-operatively (eg intensive treatment unit), together with specialist staffing, can probably only be provided effectively and economically in hospital centres. ${ }^{7}$ Equally, the Royal College of Surgeons has issued a series of recommendations regarding specialist trauma centres. ${ }^{8}$ Evidence has shown that inclusive regional trauma systems combined with the designation of high volume major trauma centres can reduce mortality by as much as $40 \%$.

While the argument to centralise and specialise often makes good clinical and financial sense, it does have an inherent tension that could make care increasingly suboptimal and costly in the future. As a former chief executive of the University Hospital Birmingham NHS Foundation Trust, I can testify to the fact that too many specialist centres operating within the same hospital building can create inefficiencies as a number of competing fiefdoms battle for resources and strive to protect, or develop, dedicated facilities which are sometimes utilised inefficiently, for example, operating theatres, medical equipment, and inpatient wards. As specialists demand more resources, care often becomes more fragmented and patient 'flow' is compromised because the holistic needs of the patients are ignored. It is also the case that a traditional hospital contains many aspects of social, primary, community, secondary and tertiary care all imperfectly existing under the same roof. So, rather than specialisation being one element of a NHS trusts strategy, it becomes the defining feature which forces cost pressures into the system as staff are overqualified for some basic care needs with resultant higher wage costs. In this scenario, unit costs per output are unnecessarily inflated and, left unchecked, ultimately lead to rationing and waiting lists.

In his book The innovator's prescription, Clayton Christensen argues that the modern day healthcare is out of date and an historical anachronism because it has been frozen into two business models - the general hospital and physician's primary care practice - that are no longer fit for purpose. ${ }^{9}$ He argues that 'the lack of business model innovation in the healthcare industry is the reason healthcare is unaffordable' and calls for massive disruptive innovation in the 21st century. As chair of KPMG's global health practice, I have noticed how some countries in the East are now pursuing a different path rather than imitating the West.

Further, specialisation is not always appropriate for patient and population needs. Our ageing population will create a need for much better general, community and social care that is properly coordinated. In the UK, the proportion of people aged 65 and over is projected to increase from 16\% in 2008 to $23 \%$ by $2022 .{ }^{11}$ We can all, on average, expect to live an extra year of life every five years and our current system of specialist care is not fit to cope with the new models that an ageing population will demand. For example, a recent survey from the Royal College of Physicians found that nearly three-quarters of all hospitals had no cover from consultant physicians specialising in acute medicine over the weekend. ${ }^{12}$ Ironically, our inability to cope with these new health and social care pressures will reduce the ability of specialists to practice, as beds and other resources are utilised by unselected medical emergencies which often present as a result of poor integration between primary and secondary care.

Equally, the rapid growth of patients presenting with longterm conditions threatens to challenge the conventional wisdom on hospital-based, specialist care. Evidence from Kaiser Permanente, based in the USA, suggests that integrated models of care can produce better patient results, less dependency on hospital-based care and improved utilisation of resources at lower or comparable costs. ${ }^{13}$ Sophisticated IT systems, coupled with good protocol based care management tools, help specialists and generalists care for the over-whelming proportion of patients with chronic conditions. Effective patient stratification and segmentation enables patients considered 'high risk' to be actively managed between community nurses and GPs, supported by an increasing range of telehealth and telecare devices which provide early warning systems and promote independent living. The approach taken by Kaiser Permanente is based on the 'chronic care model'. Kaiser focuses on integrating organisations and disciplines. Doctors from primary and secondary care share the same budget and function within multispecialty centres which also house nurses, pharmacists, laboratory technicians, radiology staff and others. People with long-term conditions are stratified according to need, with intensive management targeted at those at highest risk. 
Given that $80 \%$ of all GP consultations and $66 \%$ of all emergency hospital admissions in the UK are related to patients with long-term conditions, it is high time that our models of care and operating business models change. They do not reflect population or patient needs, are bedded in 16th century models of design and are increasingly suboptimal in terms of both their efficiency and effectiveness. As Christensen notes 'the healthcare system has trapped many disruption-enabling technologies in high cost institutions that have conflated two or three business models under the same roof.' ${ }^{9}$ Health systems that design new services with new disruptive innovation are likely to dominate the world and its future global competitiveness.

So, what does this mean for specialists and generalists alike? It means that new integrated care organisations should be created, which are owned by clinical chambers of generalists, specialists and other staff. They should be formed independently of the means and assets of production - typically the hospital - and integrated through the pathways of care and disease-specific conditions that cross traditional primary/secondary care boundaries. They should be enabled by very smart information technology which is used to inform, and increasingly deliver, care in the future. Like a professional services firm managed and owned by its partners (solicitors, barristers and accountants have been organised like this for centuries), clinical chambers would provide care services in a variety of places that were both patient and financially friendly. They would be fleet of foot, highly innovative and hold individual partner's clinical performance to account. They would negotiate prices for the facilities they utilise and would have a better risk and reward structure as they receive capitation payments for the population under their collective care. They would be held to account by the commissioner.

As the debate on the next structural change for the NHS rages (which, relatively speaking, is inconsequential compared to the care challenges we face), it is worth remembering that we need both generalists and specialists but only their collective power, when combined, can address the health issues that the UK will face for the next century. Bold, disruptive thinking by leading clinicians can bring this about, but courage and lateral thinking is needed more than ever before.

\section{References}

1 http://jasonpriem.com/2010/10/medline-literature-growth-chart

2 House of Lords Science and Technology Committee Report Volume I: Report, 2009.

3 Myocardial Ischaemia National Audit Project [MINAP]. How the NHS cares for patients with heart attack, ninth public report. London: Royal College of Physicians, 2010.

4 Hannan EL. The relation between volume and outcome in health care. New Engl J Med 2009;340.

5 Ewart $\mathrm{H}$. The relation between volume and outcome in paediatric cardiac surgery. Public Health Resource Unit - A Literature Review for the National Specialised Commissioning Group. 2009.

6 Porter M. Maximising quality, minimising cost: the concept of value for money in healthcare and the importance of clinical leadership. Monitor and UCL Partners national conference, January 2011.

7 Joint Committee of Primary Care Trusts. Safe and sustainable - review of children's congenital cardiac services in England. London: Department of Health, 2011.

8 Royal College of Surgeons. General paediatric surgery: survey of service provision in district general hospitals in England. London: RCS, 2010.

9 Christensen CM. The innovator's prescription. New York: McGraw Hill, 2009.

10 www.statistics.gov.uk

11 Royal College of Physicians. National survey of medical admissions. London: RCP, 2010.

12 Crookes PGR, Brown A. Becoming part of a multidisciplinary healthcare team. In: Daly J, Speedy S, Jackson D. Contexts of nursing. 2006.

Address for correspondence: Dr M Britnell, KPMG, 15 Canada Square, London E14 5GL.

Email: Mark.Britnell@KPMG.co.uk 